\title{
Der Zusammenhang von Chlorhexidin und Metallomatrixproteinasen
}

\begin{abstract}
Klassischerweise wird die Ursache der Dentinerweichung bei der Karies in der Säureproduktion während des Bakterienstoffwechsels gesehen. Ein weiterer, wichtiger Faktor für die Erweichung des Dentins sind jedoch auch sogenannte Metallomatrixproteinasen (MMPs), die zum ersten Mal 1962 beschrieben wurden. Sie sind am natürlichen Remodellingprozess des Knochens beteiligt, indem sie Kollagen mittels Hydrolyse lösen.
\end{abstract}

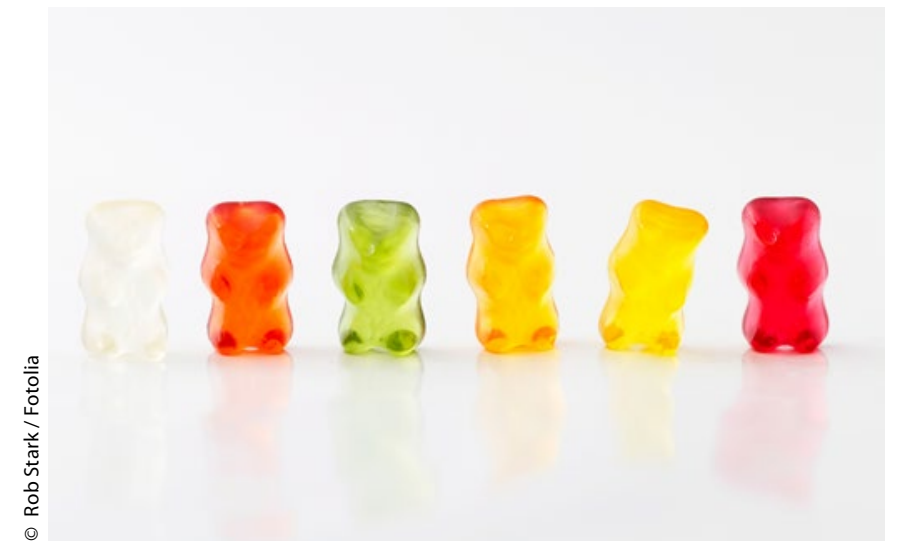

Im Dentin sind 23 verschiedene Subtypen der MMPs beschrieben worden. In saurem Milieu unter einem $\mathrm{pH}$-Wert von 4,5 steigt die Enzymaktivität, so dass die Erweichung des Dentins unter bakteriellem Säureeinfluss noch verstärkt wird. Daher konnte gezeigt werden, dass eine MMP-Inhibition eine Erosion des Dentins verhindern kann und dabei den Fluoriden sogar überlegen ist.

Neben der pathophysiologischen Bedeutung der MMPs bei der Kariesgenese spielen diese Proteine eine große Rolle bei der Spaltbildung von Kompositfüllungen:

Um die Degradierung der Hybridschicht zwischen Dentin und Komposit zu vermeiden, muss entweder das Kollagengerüst vollständig mit Bonding infiltriert oder die Degradierung des Kollagens durch die MMPs verhindert werden. Mit dem Zusatz von Chlorhexidin (CHX) zur Phosphorsäure kann dies erreicht werden.

CHX agiert als unspezifischer Inhibitor, indem es die dreidimensionale Struktur der MMPs verändert und die Konzentration von $\mathrm{Ca}^{2+}$ und $\mathrm{Zn}^{2+}$ vermindert, die für die Enzymfunktion notwendig sind. In vitro ist eine CHX-Konzentration von 0,02 Prozent ausreichend. Wegen der positiven Ladung von CHX verbleibt es für längere Zeit am Ort der Applikation. Die klini- schen Applikationen reichen von CHX zwei Prozent für 60s zu CHX 0,02 Prozent für 15s. Die Anwendung sollte immer als wässrige Lösung erfolgen. Die CHX-Mundspüllösungen enthalten oft Konservierungsstoffe, die den Bondingprozess behindern. Sie sollten daher nicht angewandt werden.

Reine, wässrige CHX-Lösungen können von der Apotheke bezogen werden. Die CHX-Lösung sollte nach dem Ätzvorgang mit einem Schaumstoffpellet aufgebracht und das Bonding nach dem Trocknen infiltriert werden. Eine Spülung mit Wasser sollte vermieden werden, Alkohol hingegen hat keinen negativen Einfluss auf CHX. Die initiale Adhäsivkraft des Bondings wird durch CHX nicht beeinflusst und auf längere Zeit sogar verstärkt. Der längste Nachbeobachtungszeitraum betrug in den vorliegenden Studien zwei Jahre.

Auch wenn CHX das sogenannte Nanoleakage nicht vollständig verhindern kann, so trägt es doch zur dauerhaften Stabilität der Hybridschicht bei.

Perspektivisch kommen neben CHX auch andere Enzyminhibitoren als Ätz- oder Bondingzusatz in Frage: EDTA (Ethylendiamintetraessigsäure), BAC (Benzalkoniumchlorid), PVPA (Polyvinylphosphonsäure) und Galadin. Andere Ansätze verfolgen die elektrische Infiltration des Dentins, um die Hybridschicht zu stabilisieren und die Diffusionsdiskrepanz von Säure und Bonding zu eliminieren. Zudem wird mit sogenannten Crosslinking-Substanzen versucht, das Kollagen zu stabilisieren.

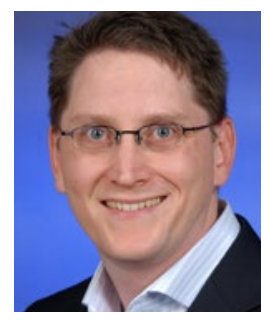

Prof. Dr. Dr. Felix P. Koch

Autor des Wissenschaftlichen Infodienstes, Lehrbeauftragter der Uniklinik Frankfurt, Praxisklinik für Mund-, Kiefer- und plastische Gesichtschirurgie in Wiesbaden Mail:wid@fvdz.de 